\title{
Detecting heterogeneity in and between breast cancer cell lines
}

Yang Shen ${ }^{1}$, B. U. Sebastian Schmidt ${ }^{1}$, Hans Kubitschke², Erik W. Morawetz ${ }^{2}$, Benjamin Wolf ${ }^{3}$, Josef A. Käs ${ }^{2}$ and Wolfgang Losert ${ }^{1 *}$ (D)

\footnotetext{
* Correspondence: wlosert@umd. edu

${ }^{1}$ Institute for Physical Science and Technology, University of Maryland, College Park, MD 20742, USA Full list of author information is available at the end of the article
}

\begin{abstract}
Background: Cellular heterogeneity in tumor cells is a well-established phenomenon. Genetic and phenotypic cell-to-cell variability have been observed in numerous studies both within the same type of cancer cells and across different types of cancers. Another known fact for metastatic tumor cells is that they tend to be softer than their normal or non-metastatic counterparts. However, the heterogeneity of mechanical properties in tumor cells are not widely studied.

Results: Here we analyzed single-cell optical stretcher data with machine learning algorithms on three different breast tumor cell lines and show that similar heterogeneity can also be seen in mechanical properties of cells both within and between breast tumor cell lines. We identified two clusters within MDA-MB231 cells, with cells in one cluster being softer than in the other. In addition, we show that MDA-MB-231 cells and MDA-MB-436 cells which are both epithelial breast cancer cell lines with a mesenchymal-like phenotype derived from metastatic cancers are mechanically more different from each other than from non-malignant epithelial MCF-10A cells.
\end{abstract}

Conclusion: Since stiffness of tumor cells can be an indicator of metastatic potential, this result suggests that metastatic abilities could vary within the same monoclonal tumor cell line.

Keywords: Cancer, Heterogeneity, Single-cell, MDA-MB-231, MCF-10A, MDA-MB-436

\section{$\triangle B M C$}

\section{Background}

Recognized as early as 1958 (Huxley 1958), genetic heterogeneity is a well-established phenomenon in tumor cells, especially during metastatic stages (Torres et al. 2007; Park et al. 2010; Patel et al. 2014; Alizadeh et al. 2015). Studies have shown that cells from a single cancer typically contain multiple genetically distinct subgroups (Cleary et al. 2014; Meacham and Morrison 2013; Gay et al. 2016; Marusyk and Polyak 2010). Such high level of heterogeneity contributes to the reason why cancer is hard to cure (McGranahan and Swanton 2017; Mann et al. 2016; Koren and Bentires-Alj 2015). However, to-date the reason and extent of tumor cell heterogeneity is still not wellunderstood (Alizadeh et al. 2015). Two main theories have been proposed to explain the origin of tumor cell heterogeneity: the existence of cancer stem cells (Magee et al. 2012) and clonal evolution (McGranahan and Swanton 2017). These two theories try to explain the heterogeneity in ecological and evolutional aspects, respectively, and

(c) The Author(s). 2020 Open Access This article is distributed under the terms of the Creative Commons Attribution 4.0 International License (http://creativecommons.org/licenses/by/4.0/), which permits unrestricted use, distribution, and reproduction in any medium, provided you give appropriate credit to the original author(s) and the source, provide a link to the Creative Commons license, and indicate if changes were made. 
evidence exists for each theory (Shackleton et al. 2009). Furthermore, new insight in gene regulatory networks provides a framework for explaining the broad heterogeneity without the need of excessive mutational activity (Huang 2012a; Huang 2013; Huang 2012b). Variations in gene expression lead to molecular variations which in turn affect cellular shape and function.

Another well-established phenomenon associated with tumors are changes in cellular stiffness. Cells actively structure and regulate the different elements of the cytoskeleton, the main contributor of cellular stiffness and compliance (Huber et al. 2013). In fact, different components of the cytoskeleton contribute to different structural and mechanical tasks, e.g. actin contributes to cell elasticity in response to small strains while microtubules affect responses to large strains (Lautenschlager et al. 2009; Kubitschke et al. 2017). The mechanics of cells has been studied with multiple experimental tools (Kubitschke et al. 2018; Pawlizak et al. 2015), including atomic force microscopy (AFM) (Hayashi and Iwata 2015), quantitative deformability cytometry (q-DC) (Nyberg et al. 2017), real-time deformability cytometry (Mietke et al. 2015; Otto et al. 2015), microfluidic optical cell stretchers (Farzbod and Moon 2018), and hydrodynamic flow stretchers (Dudani et al. 2013; Gossett et al. 2012). Since metastasis is responsible for more than $90 \%$ of cancer fatality (Wirtz et al. 2011; Mehlen and Puisieux 2006; Taketo 2011), great effort has been made to study the mechanical properties of metastatic tumor cells and to understand how mechanical properties of tumor cells affect their metastatic ability. A number of studies have found that metastatic tumor cells are softer than their non-metastatic counterparts as well as normal cells (Lekka et al. 2012; Plodinec et al. 2012; Swaminathan et al. 2011). In addition, studies have suggested the potential of using mechanical properties as a biomarker of metastasis (Xu et al. 2012) and for cancer diagnosis (Remmerbach et al. 2009).

In this paper we take first steps to link these two phenotypes of metastatic tumor cells - changes in cell heterogeneity and cell stiffness. Though most cell mechanics studies are carried out at the single-cell level, analysis and interpretation of data is generally confined to averages, thus omitting heterogeneity as an important aspect of the metastatic phenotype. Prior work (Plodinec et al. 2012; Kiessling et al. 2013) has yielded important hints that mechanical properties are in fact heterogeneous - the measured distributions for the viscoelastic properties of cells, even in a single cell line, are not Gaussian indicating that various mechanical phenotypes are present, for instance, represented by outliers of the usual long-tailed distributions.

In this paper, we use a microfluidic optical cell stretcher to measure and contrast mechanical properties of single cells from three epithelial cell lines: MCF-10A, MDA-MB-231 and MDA-MB-436, and we use the heterogeneity of the cell mechanical properties of each cell line to contrast the different phenotypes. These three cell lines represent a well-established breast cancer cell panel. MCF-10A is a non-tumorigenic epithelial cell line while MDA-MB-436 and MDA-MB-231 are breast carcinoma cell lines with a mesenchymal-like metastatic phenotype. With single cell data analysis, we show that heterogeneity of cellular stiffness exists both within and between cell lines. In particular, we observe two groups of MDA-MB-231 cells. Cells in one of the groups are significantly softer than cells in the other. In addition, we find that although MDA-MB-231 and MDA-MB-436 are both triple-negative breast cancer cell lines (i.e. they do not express estrogen receptors, progesterone receptors nor human epidermal growth factor receptor [HER]2) with metastatic tendency, they are rather distinct from each other compared to the nonmalignant cell line MCF-10A. 


\section{Results}

We used a Microfluidic Optical Cell Stretcher to mechanically stretch individual cells from our breast cancer panel of cell lines and measure their stiffness (Kiessling et al. 2013; Lincoln et al. 2007a). Cells in suspension are not stimulated by their environment, and thus their cortical tension represents the cells' mechanical "ground state". Suspended single cells were trapped for $1 \mathrm{~s}$ and subsequently stretched for $2 \mathrm{~s}$ and then relaxed in trapping condition for another $2 \mathrm{~s}$ (Fig. 1). Images of cells were taken at the rate of 30 frames per second, and the length of the long axis was measured in each frame for each individual cell. In this paper, we use only two mechanical features calculated from these measurements: 1 . Relative long axis deformation at the end of stretch (Deformation EOS), and 2. Relative long axis deformation after $2 \mathrm{~s}$ of relaxation (Relaxation EOE) (Fig. 1). The value of EOS is inversely proportional to the Young's modulus, where higher EOS value indicates lower Young's modulus (easier to stretch). On the other hand, EOE is a measure of the ability of a cell to restore its shape, where higher absolute value of EOE suggests greater ability to restore the original cell shape. The end-of-experiment deformation (EOE) can also be interpreted as the degree of cell plasticity of the cell under a given applied load or strain. This plasticity is in principle a coarse-grained property which contains contributions of the actin, microtubule and intermediate filament network (Kubitschke et al. 2017). Since both EOE and EOS are linear measurements, a two-fold change in the observed deformation corresponds to a two-fold change in elastic modulus.

In prior work where the mechanical measurements were parameterized by over 50 metrics, we identified deformation and relaxation as important independent

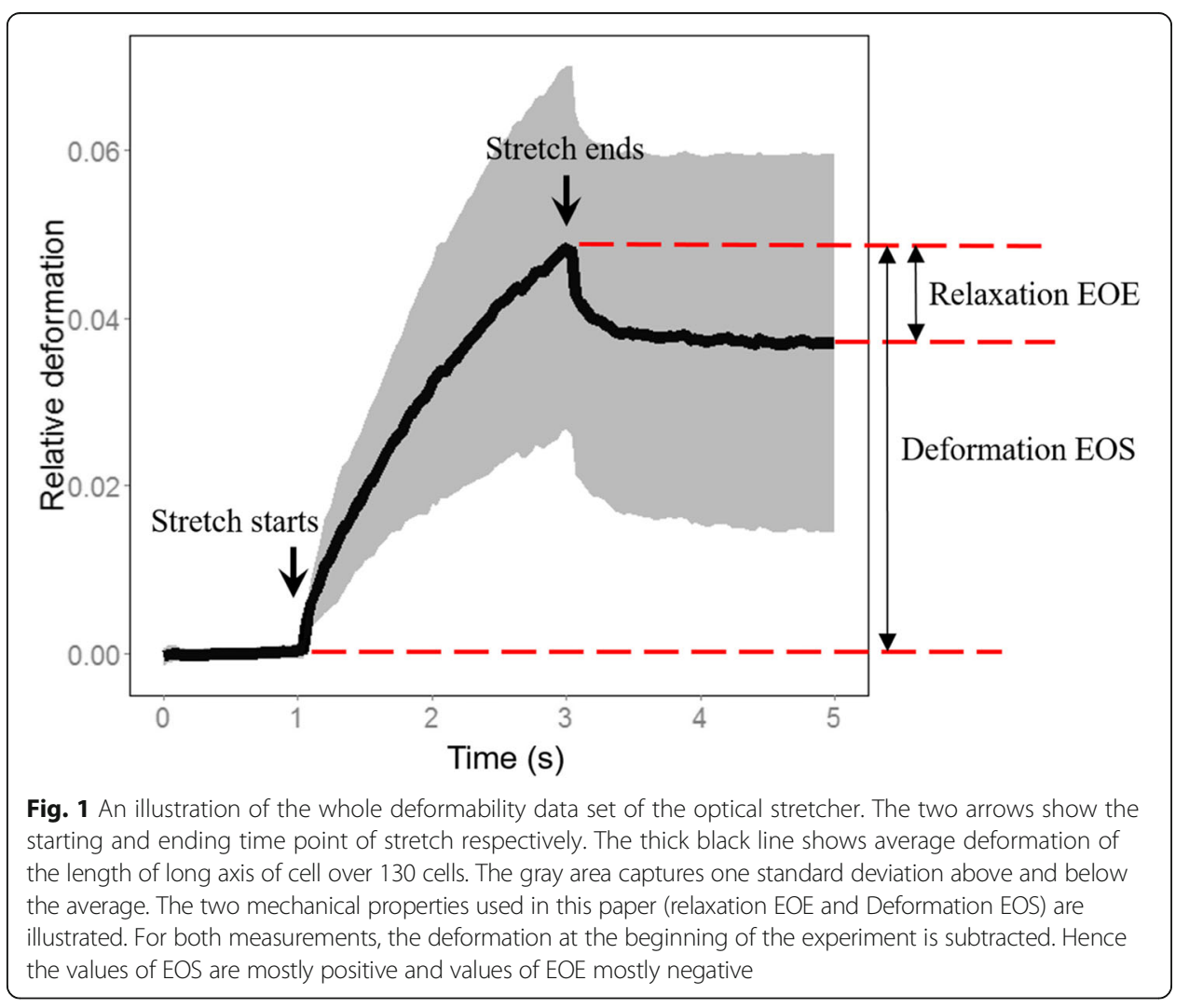


determinants of cell mechanics (Kiessling et al. 2013). Together, these two features give a good estimation of the elastic property of a single cell.

Using this technique, we measured cells from our breast cancer cell panel used to study the EMT. MCF-10A is a non-tumorigenic breast epithelial cell line which is used as a control cell line. MDA-MB-231 and MDA-MB-436 are both triple negative breast cancer cell lines that are epithelial in nature. Both have metastatic potential, with MDA-MB-231 considered more aggressive than MDA-MB-436 (Bianchini et al. 2016).

\section{Two subgroups observed in MDA-MB-231 cells}

We first identified two subgroups within MDA-MB-231 cells. One subgroup (cluster 2, Fig. 2) exhibited higher deformations at the end of stretch (EOS) and higher absolute values of relaxation at the end of experiment (EOE) than the other subgroup (cluster 1) (Fig. 2). Higher absolute values of both EOS and EOE indicate that cells in cluster 2 are softer and more elastic (easier to stretch and easier to restore original shape) compared to cluster 1, which overlaps with MDA-MB-436 and MCF-10A cells (Fig. 3a).

\section{The more elastic group does not exist in MDA-MB-231 cells labeled for E-cadherin}

Cadherins are responsible for cell-cell binding. E-cadherins are expressed in normal epithelial cells, while in mesenchymal carcinoma cells it is mainly $\mathrm{N}$-cadherins. In our experiments, we also measured mechanical properties of MDA-MB-231 cells that were labeled with E-cadherin antibodies in order to activate extracellular binding sites. Since this is a mesenchymal-like cell line we found a low level of E-cadherin expression, as has also been quantified elsewhere (Pawlizak et al. 2015). In spite of the low expression levels, we observed a different stretching and relaxation behavior in the E-cadherin labeled and non-labeled MDA-MB-231 cells. E-cadherin labeled MDA-MB-231 cells only formed one cluster instead of the two clusters observed in unlabeled MDA-MB-231 cells. The labeled 231 cells overlap with the less elastic and less relaxing subgroup of

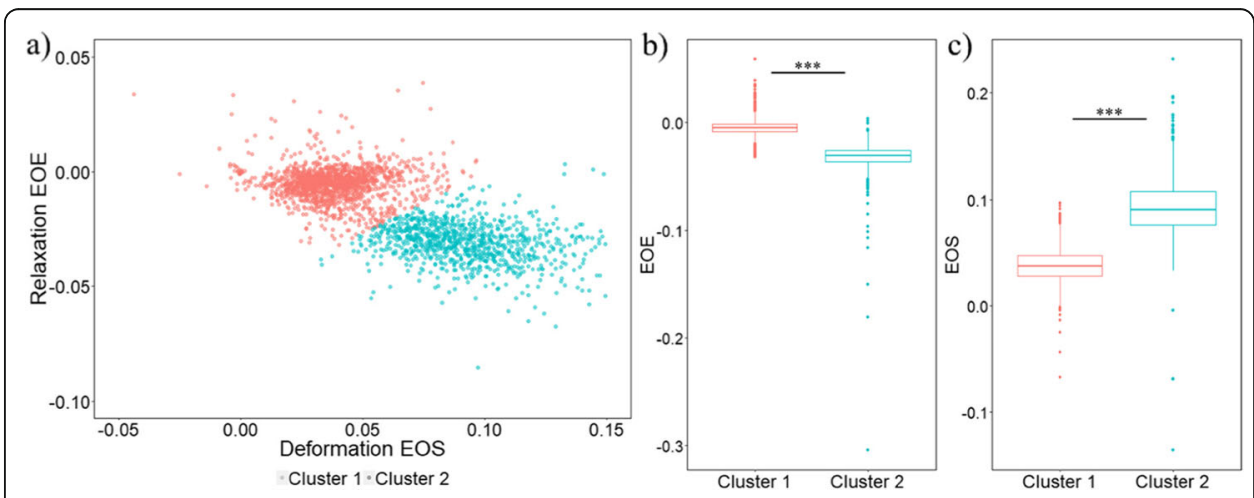

Fig. 2 Two clusters of MDA-MB-231 cells are observed. a Scatterplot of Relaxation EOE vs Deformation EOS for MDA-MB-231 cells. The two subgroups are identified with the k-means clustering algorithm and labeled by different colors (red: cluster 1, blue: cluster 2). Negative EOS values in the plot can have two causes: first, strongly rotating cells that influence the shape detection; second, active contractions under force activation (data not shown). Similarly, a positive EOE values can indicate a strongly rotating cells or continued deformation during the relaxation phase. $\mathbf{b}$ Boxplot comparing relaxation at the end of experiment between cluster 1 and cluster 2 of MDA-MB-231 cells ( $p$ value $<0.001$ ). c Boxplot comparing deformation at the end of stretch between the two subgroups of MDA-MB-231 cells ( $p$ value $<0.001$ ) 

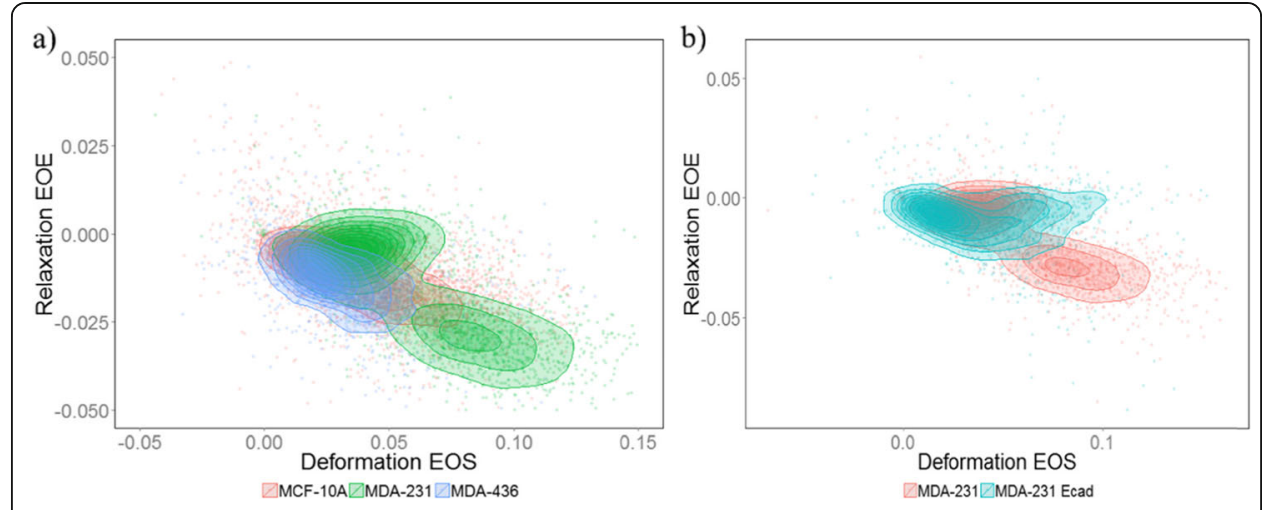

Fig. 3 MCF-10A, MDA-MB-436 and E-cadherin labeled MDA-MB-231 cells all overlap with cluster 1 (the less elastic group) in unlabeled MDA-MB-231 cells. a Scatterplot of Relaxation EOE vs Deformation EOE for MCF$10 A$ (red), MDA-MB-231 (green) and MDA-MB-436 (blue) cells. b Scatterplot of Relaxation EOE vs Deformation EOE for E-cadherin labeled (blue) and unlabeled (red) MDA-MB-231 cells

MDA-MB-231 cells (cluster 1, Fig. 3b). Activation of the E-cadherin receptor by binding of the antibody leads to cadherin clustering and E-cadherin binding to the actin cortex, which upregulates the actin polymerization and cross-linking of the cytoskeleton (Perez-Moreno and Fuchs 2006). The decrease in deformation found in cluster 1 cells compared to cluster 2 cells is consistent with this change in mechanics due to Ecadherin activation since the elastic storage modulus strongly depends on crosslinking density and dynamics (Gardel et al. 2004; Lieleg et al. 2010; Strehle et al. 2011; Schnauß et al. 2016). In addition, the decreased cell relaxation of the cluster 1 subpopulation could also be explained with upregulated actin nucleation and aggregation while a destabilization of the microtubular cytoskeletal backbone may further result in a lack of relaxation and increased plasticity (Kubitschke et al. 2017).

MDA-MB-231 and MDA-MB-436 cells are more different from each other than from MCF$10 \mathrm{~A}$ cells

While we showed above that cluster 1 of MDA-MB-231 cells greatly overlaps with MCF-10A and MDA-MB-436 cells, these three cell lines may still be separable at the single cell level. Since both MDA-MB-436 and MDA-MB-231 cell lines have a malignant mesenchymal-like phenotype, it is reasonable to expect they would be more similar to each other comparing to the epithelial-like MCF-10A cell line. To separate the cell phenotypes, we applied a k nearest neighbors (k-NN) algorithm for a pairwise classification of the three phenotypes. We first divided the cells into two groups: train and test. Phenotype labels were provided for cells in the training group but not for the test group. Then, given the position of a single cell in the test group, k-NN identifies its nearest $\mathrm{k}$ neighbors within the training group. The $\mathrm{k}$ neighbors then take a "vote" with their phenotype, and the cell from test group is assigned to the phenotype that has the highest number of votes. After classification, we calculate the sensitivity (true positive rate), specificity (true negative rate), and F1 score (a measure of classification result, the higher the score the better the classification; the maximum F1 score is 1) for each pair of classification. We found that classification between MCF-10A and MDA-MB-436 cells has the lowest sensitivity, specificity and F1 score regardless of the value of $k$ 
(green line in Fig. 4). On the other hand, classification between cluster 1 of MDA-MB231 and MDA-MB-436 cells had the highest level of F1 scores (blue line in Fig. 4c) which was even higher than the classification between MCF-10A and MDA-MB-231 cells (red line in Fig. 4c) for most values of k. Similar results were obtained with a different classification algorithm (SVM), where the classification between MDA-MB-231 and MDA-MB-436 cells also had the highest F1 value (Table 1). SVM takes a different approach in classification and aims to find the linear plane that best separates two groups to classify. In addition, when all four phenotypes were classified simultaneously, MDA-MB-436 cells were less likely to be miss-classified as MDA-MB-231 cells than as MCF-10A cells and vice versa (Fig. 5). Together, these results suggest that cells in cluster 1 of MDA-MB-231 are more different from MDA-MB-436 cells than from MCF10A cells despite that MDA cells are phenotypically considered to be mesenchymal-like and MCF cells to be epithelial.

\section{Discussion}

Mechanical properties of tumors cells may be important markers for the metastatic potential of tumors. Studies have shown that metastatic tumor cells are on average softer than non-metastatic ones (Xu et al. 2012; Guck et al. 2005; Fritsch et al. 2010; Alibert et al. 2017; Mierke 2015). In this paper, we illustrate the heterogeneity of tumor cell stiffness both within and between cell lines. Based only on mechanical properties, we show that there are two distinct clusters within MDA-MB-231 cells. Cluster 1 greatly overlaps with MCF-10A and MDA-MB-436 cells, while cells in cluster 2 are softer and more elastic (easier to deform and restore to original shape). In addition, we found that the two malignant epithelial cell lines, MDA-231 and MDA-436, are more distinct from each other in their mechanical phenotype than from the non-tumorigenic MCF-10A cell line.

Our findings of mechanical heterogeneity within the MDA-MB-231 cell line complement prior findings indicating that the molecular single cell characteristics of MDAMB-231 cells are also heterogeneous. For example, it has been shown that there are two distinct subgroups of MDA-MB-231 cells which differ significantly in the cell surface density of various cytokine receptors (CCR5, CXCR3, CXCR1) (Norton et al.

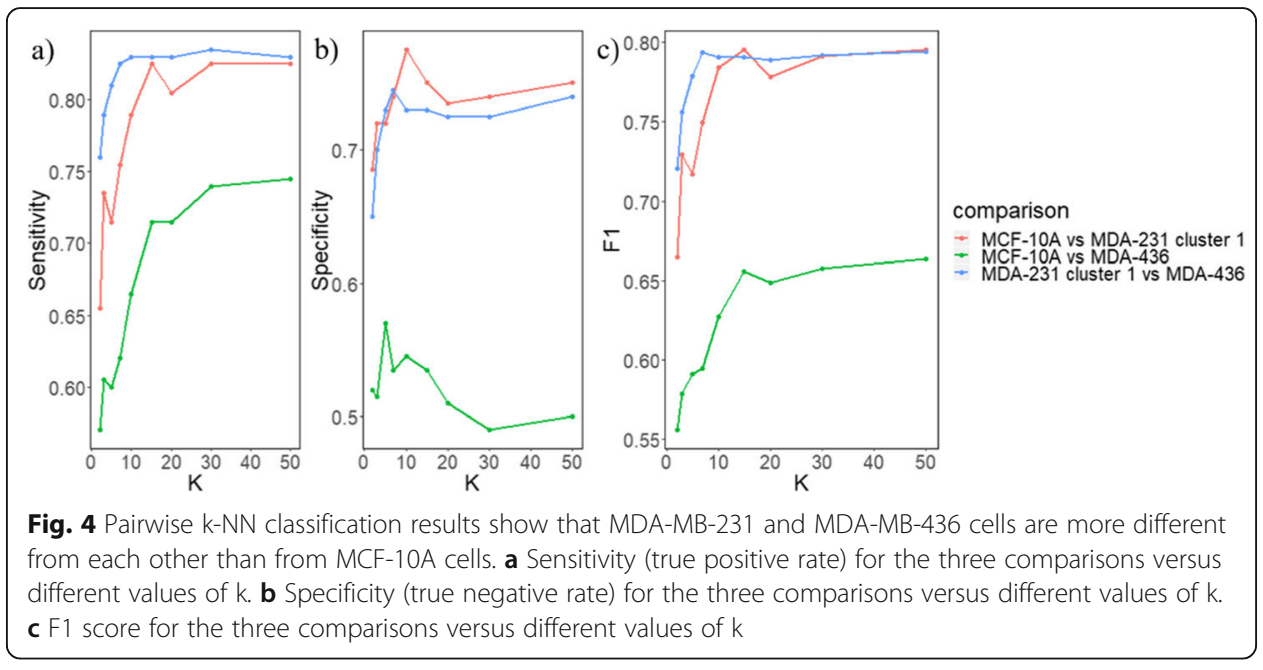


Table 1 Pairwise classification results by support vector machine (SVM)

\begin{tabular}{llll}
\hline & Sensitivity & Specificity & F1 \\
\hline MCF-10A (positive) vs MDA-MB-231 cluster 1 (negative) & 0.71 & 0.72 & 0.71 \\
MCF-10A (positive) vs MDA-MB-436 (negative) & 0.58 & 0.66 & 0.60 \\
MDA-MB-231 cluster 1 (positive) vs MDA-MB-436 (negative) & 0.74 & 0.79 & 0.76 \\
\hline
\end{tabular}

2015). In particular, CXCR3 was found to be overexpressed in metastatic tumor cells, and drugs targeting CXCR3 decreased tumor cell migration (Zhu et al. 2015). To link our observations of mechanical heterogeneity with molecular heterogeneity directly, future studies can combine the optical stretching with fluorescence imaging.

We also identified heterogeneity among different triple negative breast cancer (TNBC) cell lines, i.e. we found that MDA-MB-231 and MDA-MB-436 cells are quite distinct from each other, even more so than from the non-tumorigenic MCF-10A cell line. This finding is consistent with the perspective of the classical clonal evolution model, assuming the epigenetic and (more importantly) the phenotypic characteristics of normal breast tissue are similar among all women. Thus, both patients from which the MDA-MB-231 and MDA-MB-436 cell lines are derived, had initially breast tissue which is very similar to the MCF-10A tissue. From this healthy starting population of cells, different paths can be taken to reach a metastatic phenotype. In fact, an extensive gene similarity analysis based on The Cancer Genome Atlas (TCGN) revealed that on average approx. $40 \%$ of tumors of a given site, e.g. breast cancers, are likely genetically closer to tumors from other sites than to tumors of the same origin (Heim et al. 2014; Andor et al. 2016). It seems actually unlikely that two completely different patients accumulate the exact same cancer cell phenotype with the same optical stretching characteristics.

In addition, our findings may have important clinical implications. Patients with triple negative breast cancer are currently considered to have a very poor prognosis (Bianchini

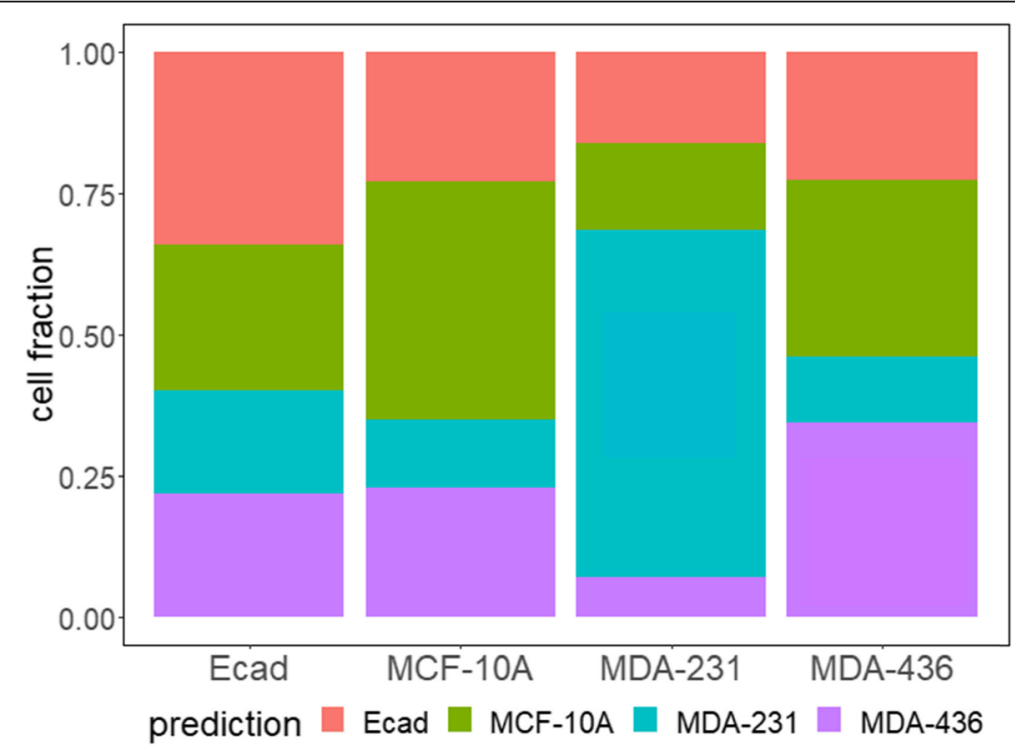

Fig. 5 k-NN classification results of E-cadherin labeled MDA-MB-231 cells (Ecad), MCF-10A cells, cluster 1 in MDA-MB-231 cells and MDA-MB-436 cells, with $k=10$ 
et al. 2016; Lehmann and Pietenpol 2014; Denkert et al. 2017). However, there has been an emerging trend to regard TNBC as a heterogeneous group of patients with varying prognosis (Bianchini et al. 2016; Lehmann and Pietenpol 2014; Denkert et al. 2017). Furthermore, TNBCs can have very different molecular characteristics, potentially rendering some tumors more suitable to targeted therapies (Bianchini et al. 2016; Lehmann and Pietenpol 2014; Denkert et al. 2017). It is of paramount clinical importance to identify those patients. The present data is exciting in that it shows that two TNBC cell lines (which would be put into one prognostic basket clinically) are indeed very different. It is intriguing to speculate whether optical stretching analysis could be used to differentiate those TNBC cases with a better prognosis (i.e. a lower rate of relapse and distant metastasis) from those with a worse prognosis.

Moreover, our findings on the inter-cell-line heterogeneity is an indication that average based analysis methods could oversimplify tumor cell data. For example, MCF-10A, MDA-MB-436 and cluster 1 of MDA-MB-231 cells are mechanically similar to each other with probably minor difference in the average values (Fig. 3a). However, when classified with a more sophisticated algorithm like k-NN, reasonably good classification accuracy can be achieved. That is to say, even though cells from the three cell lines overlap on average, locally cells from a certain cell line are closer to cells from the same cell line than from other cell lines.

Lastly, our studies of E-cadherin labeled MDA-MB-231 cells reveal that antibody labeling can alter the mechanical phenotype significantly. We reason that this is because binding of the antibody to the E-cadherin receptor simulates cell-cell binding, which causes cadherin clustering and stimulates the actin cortex bound to cadherin. This is a good example of how antibody labeling may change the properties of cells, and how antibodies could provide insights into the changes in cancer cell behavior in response to their tumor microenvironment. Further experiments are needed to validate and provide molecular evidence for the role of E-cadherin antibody treatment in altering the mechanical phenotype of MDA-MB-231 cells.

\section{Conclusion}

In conclusion, we illustrated heterogeneity in cellular mechanical properties within and between cell lines. Future studies should examine how changes in chemokine receptor expression correlate with tumor cell stiffness. Additional investigations are needed to determine how mechanical properties of cancer cells could help identify distinct prognostic subgroups of triple negative breast cancer patients.

\section{Methods and materials}

\section{Experimental procedures}

The general setup of the optical stretcher (OS) is described in (Lincoln et al. 2007b) with additional improvements to the microfluidics, the computer-controlled stretching processes, and the thermally controlled stage described in detail in (Lincoln et al. 2007b; Guck et al. 2001; Schmidt et al. 2015). The mechanical properties of cells were determined by guiding the cell suspension into the automated microfluidic OS where single cells are consecutively trapped and stretched. The cells are trapped at $100 \mathrm{~mW}$ for $1 \mathrm{~s}$ and the cell radius along the laser axis is determined. The cell is afterwards 
stretched at $875 \mathrm{~mW}$ for $2 \mathrm{~s}$. The cells are allowed to relax for $2 \mathrm{~s}$ after stress cessation. A microscope-mounted camera takes images at 30 frames per second during the whole stretching process. Afterwards, an edge detection algorithm is used to extract cell shape and cell parameters and to sort out pathological cell (e.g. dead cells).

\section{Cell culture and medium}

MCF-10A cells (Cat.No. CRL-10317, ATCC) were cultured in DMEM/Ham's F12 medium containing l-glutamine (Cat.No. FG 4815, Biochrom) supplemented with 5\% horse serum (Cat.No. 12449C, SAFC), $20 \mathrm{ng} / \mathrm{ml}$ human epidermal growth factor (Cat.No. E9644, Sigma-Aldrich), $10 \mu \mathrm{g} / \mathrm{ml}$ insulin (Cat. No.19278, Sigma-Aldrich), 100 $\mathrm{ng} / \mathrm{ml}$ cholera toxin (Cat.No. C8052, Sigma-Aldrich), $500 \mathrm{ng} / \mathrm{ml}$ hydrocortisone (Cat.No. H0888, Sigma-Aldrich) and $100 \mathrm{U} / \mathrm{ml}$ penicillin/streptomycin (Cat.No. A 2213, Biochrom).

MDA-MB-231 and MDA-MB-436 cells were cultured in DMEM containing 4.5 g/l glucose, l-glutamine (Cat.No. FG 0435, Biochrom) supplemented with $10 \%$ fetal bovine serum (Cat.No. S 0615, Biochrom) and $100 \mathrm{U} / \mathrm{ml}$ penicillin/streptomycin.

All cell lines were incubated at $37^{\circ} \mathrm{C}$ in a $95 \%$ air and $5 \% \mathrm{CO} 2$ atmosphere. The culture medium was changed every 2 to 3 days and cells were passaged every 4 to 5 days. To detach the cells, a PBS solution containing $0.025 \%(\mathrm{w} / \mathrm{v})$ trypsin and $0.011 \%(\mathrm{w} / \mathrm{v})$ EDTA (Cat.No. L 2113, Biochrom) was applied for several minutes.

\section{Data analysis}

The two clusters of MDA-MB-231 cells were identified using the kmeans() function in $R$ (version 3.0.3) with 2 centers, 1000 iterations and 50 random initial conditions. For kNN classification, 1200 cells were first randomly selected from each cell line. From the 1200 cells, 200 were randomly selected as testing set and the remaining 1000 were used as training set for each cell line. The classification was done separately for each pair of cell line using the $k n n()$ function in $\mathrm{R}$ with 8 different values of $k(2,3,5,7,10,15,20,50)$. Similarly, simultaneous classification of the three cell lines were done. After classification, a false positive rate was calculated as FPR $=$ (false positives) $/$ (false positives + true positives), and a false negative rate was calculated as $($ FNR $)=$ (false negatives) $/$ (false negatives + true negatives). Finally, pairwise support vector machine (SVM) classifications were done based on all 1200 randomly selected cells using the $k s v m()$ function with linear kernel and $C=10$ in the $R$ package kernlab. All plots were made with the ggplot2 package in $R$. The dataset is normalized to zero mean and unit variance before the aforementioned analysis.

\section{Abbreviations}

EOE: End of experiment deformation; EOS: End of stretch deformation; FNR: False negative rate; FPR: False positive rate; kNN: k nearest neighbors; OS: Optical stretcher; SVM: Support vector machine; TCGN: The Cancer Genome Atlas; TNBC: Triple negative breast cancer

\section{Acknowledgements}

Not applicable

\section{Authors' contributions}

YS analyzed data and wrote the manuscript, BUSS and BW helped interpret the data and edit the manuscript, HK and EWM carried out the experiment and edited the manuscript, WL and JAK supervised the project and edited the manuscript. All authors read and approved the final manuscript. 


\section{Funding}

This project has received funding from the European Research Council (ERC-741350/HoldCancerBack) and we acknowledge funding from the European Commission H2020-PHC-2015-two-stage in the frame of the 'FORCE' project. W.L. was partially supported by AFOSR grant FA9550-16-1-0052. Y.S. was supported by the National Institutes of Health National Eye Institute intramural research program. E.M. was supported by the European Union and the Europäischer Sozialfonds in Saxony (ESF - 100234741).

\section{Availability of data and materials}

Data is available upon request.

\section{Ethics approval and consent to participate}

Not applicable.

\section{Consent for publication}

Not applicable.

\section{Competing interests}

The authors declare that they have no competing interests.

\section{Author details}

${ }^{1}$ Institute for Physical Science and Technology, University of Maryland, College Park, MD 20742, USA. ${ }^{2}$ Peter Debye Institute for Soft Matter Physics, Leipzig University, Linnéstr. 5, 04103 Leipzig, Germany. ${ }^{3}$ Leipzig University Medical Center, Department of Obstetrics and Gynecology, Liebigstr. 20a, 04103 Leipzig, Germany.

Received: 7 June 2019 Accepted: 15 January 2020

\section{Published online: 03 February 2020}

\section{References}

Alibert C, Goud B, Manneville JB. Are cancer cells really softer than normal cells? Biol Cell. 2017;109(5):167-89.

Alizadeh AA, Aranda V, Bardelli A, Blanpain C, Bock C, Borowski C, et al. Toward understanding and exploiting tumor heterogeneity. Nat Med. 2015;21(8):846-53 Epub 2015/08/08.

Andor N, Graham TA, Jansen M, Xia LC, Aktipis CA, Petritsch C, et al. Pan-cancer analysis of the extent and consequences of intratumor heterogeneity. Nat Med. 2016;22(1):105-13 Epub 2015/12/01.

Bianchini G, Balko JM, Mayer IA, Sanders ME, Gianni L. Triple-negative breast cancer: challenges and opportunities of a heterogeneous disease. Nat Rev Clin Oncol. 2016;13(11):674-90 Epub 2016/10/19.

Cleary AS, Leonard TL, Gestl SA, Gunther EJ. Tumour cell heterogeneity maintained by cooperating subclones in Wnt-driven mammary cancers. Nature. 2014;508(7494):113-7 Epub 2014/04/04.

Denkert C, Liedtke C, Tutt A, von Minckwitz G. Molecular alterations in triple-negative breast cancer-the road to new treatment strategies. Lancet. 2017;389(10087):2430-42 Epub 2016/12/13.

Dudani JS, Gossett DR, Tse HT, Di Carlo D. Pinched-flow hydrodynamic stretching of single-cells. Lab Chip. 2013;13(18):372834 Epub 2013/07/26.

Farzbod A, Moon H. Integration of reconfigurable potentiometric electrochemical sensors into a digital microfluidic platform. Biosens Bioelectron. 2018;106:37-42 Epub 2018/02/08.

Fritsch A, Höckel M, Kiessling T, Nnetu KD, Wetzel F, Zink M, et al. Are biomechanical changes necessary for tumour progression? Nat Phys. 2010;6(10):730

Gardel M, Shin J, MacKintosh F, Mahadevan L, Matsudaira P, Weitz D. Elastic behavior of cross-linked and bundled actin networks. Science. 2004;304(5675):1301-5.

Gay L, Baker AM, Graham TA. Tumour Cell Heterogeneity. F1000Res. 2016;5 Epub 2016/03/15.

Gossett DR, Tse HT, Lee SA, Ying Y, Lindgren AG, Yang OO, et al. Hydrodynamic stretching of single cells for large population mechanical phenotyping. Proc Natl Acad Sci U S A. 2012;109(20):7630-5 Epub 2012/05/02.

Guck J, Ananthakrishnan R, Mahmood H, Moon TJ, Cunningham CC, Käs J. The optical stretcher: a novel laser tool to micromanipulate cells. Biophys J. 2001;81(2):767-84.

Guck J, Schinkinger S, Lincoln B, Wottawah F, Ebert S, Romeyke M, et al. Optical deformability as an inherent cell marker for testing malignant transformation and metastatic competence. Biophys J. 2005;88(5):3689-98.

Hayashi K, Iwata M. Stiffness of cancer cells measured with an AFM indentation method. J Mech Behav Biomed Mater. 2015; 49:105-11 Epub 2015/05/26.

Heim D, Budczies J, Stenzinger A, Treue D, Hufnagl P, Denkert C, et al. Cancer beyond organ and tissue specificity: nextgeneration-sequencing gene mutation data reveal complex genetic similarities across major cancers. Int I Cancer. 2014; 135(10):2362-9 Epub 2014/04/08.

Huang S. Tumor progression: chance and necessity in Darwinian and Lamarckian somatic (mutationless) evolution. Prog Biophys Mol Biol. 2012a;110(1):69-86 Epub 2012/05/15.

Huang $\mathrm{S}$. The molecular and mathematical basis of Waddington's epigenetic landscape: a framework for post-Darwinian biology? BioEssays. 2012b;34(2):149-57 Epub 2011/11/22

Huang S. Genetic and non-genetic instability in tumor progression: link between the fitness landscape and the epigenetic landscape of cancer cells. Cancer Metastasis Rev. 2013;32(3-4):423-48 Epub 2013/05/04.

Huber F, Schnauss J, Ronicke S, Rauch P, Muller K, Futterer C, et al. Emergent complexity of the cytoskeleton: from single filaments to tissue. Adv Phys. 2013;62(1):1-112 Epub 2013/01/01.

Huxley J. Biological aspects of cancer: Harcourt, Brace; 1958.

Kiessling TR, Herrera M, Nnetu KD, Balzer EM, Girvan M, Fritsch AW, et al. Analysis of multiple physical parameters for mechanical phenotyping of living cells. Eur Biophys J. 2013;42(5):383-94 Epub 2013/03/19. 
Koren S, Bentires-Alj M. Breast tumor heterogeneity: source of fitness, Hurdle for Therapy. Mol cell. 2015;60(4):537-46 Epub 2015/11/23.

Kubitschke H, Morawetz EW, Käs JA, Schnauß J. Physical Properties of Single Cells and Collective Behavior. In: Quantification of Biophysical Parameters in Medical Imaging. Cham: Springer; 2018. p. 89-121.

Kubitschke H, Schnauss J, Nnetu K, Warmt E, Stange R, Kaes J. Actin and microtubule networks contribute differently to cell response for small and large strains. New J Phys. 2017;19(9):093003.

Lautenschlager F, Paschke S, Schinkinger S, Bruel A, Beil M, Guck J. The regulatory role of cell mechanics for migration of differentiating myeloid cells. Proc Natl Acad Sci U S A. 2009;106(37):15696-701 Epub 2009/09/01.

Lehmann BD, Pietenpol JA. Identification and use of biomarkers in treatment strategies for triple-negative breast cancer subtypes. J Pathol. 2014;232(2):142-50.

Lekka M, Pogoda K, Gostek J, Klymenko O, Prauzner-Bechcicki S, Wiltowska-Zuber J, et al. Cancer cell recognition--mechanical phenotype. Micron. 2012;43(12):1259-66 Epub 2012/03/23.

Lieleg O, Claessens MM, Bausch AR. Structure and dynamics of cross-linked actin networks. Soft Matter. 2010;6(2):218-25.

Lincoln B, Schinkinger S, Travis K, Wottawah F, Ebert S, Sauer F, et al. Reconfigurable microfluidic integration of a dual-beam laser trap with biomedical applications. Biomed Microdevices. 2007a;9(5):703-10 Epub 2007/05/17.

Lincoln B, Wottawah F, Schinkinger S, Ebert S, Guck J. High-throughput rheological measurements with an optical stretcher. Methods Cell Biol. 2007b;83:397-423.

Magee JA, Piskounova E, Morrison SJ. Cancer stem cells: impact, heterogeneity, and uncertainty. Cancer Cell. 2012;21(3):28396 Epub 2012/03/24.

Mann KM, Newberg JY, Black MA, Jones DJ, Amaya-Manzanares F, Guzman-Rojas L, et al. Analyzing tumor heterogeneity and driver genes in single myeloid leukemia cells with SBCapSeq. Nat Biotechnol. 2016;34(9):962-72 Epub 2016/08/02.

Marusyk A, Polyak K Tumor heterogeneity: causes and consequences. Biochim Biophys Acta. 2010;1805(1):105-17 Epub 2009/11/26.

McGranahan N, Swanton C. Clonal heterogeneity and tumor evolution: past, present, and the future. Cell. 2017;168(4):613-28 Epub 2017/02/12

Meacham CE, Morrison SJ. Tumour heterogeneity and cancer cell plasticity. Nature. 2013;501(7467):328-37 Epub 2013/09/21.

Mehlen P, Puisieux A. Metastasis: a question of life or death. Nat Rev Cancer. 2006;6(6):449-58 Epub 2006/05/26.

Mierke CT. Cellular stiffness and deformability. Bristol: Physics of Cancer: IOP Publishing; 2015. p. 3-31.

Mietke A, Otto O, Girardo S, Rosendahl P, Taubenberger A, Golfier S, et al. Extracting cell stiffness from real-time deformability Cytometry: theory and experiment. Biophys J. 2015;109(10):2023-36 Epub 2015/11/21.

Norton KA, Popel AS, Pandey NB. Heterogeneity of chemokine cell-surface receptor expression in triple-negative breast cancer. Am J Cancer Res. 2015:5(4):1295-307 Epub 2015/06/24

Nyberg KD, Hu KH, Kleinman SH, Khismatullin DB, Butte MJ, Rowat AC. Quantitative deformability Cytometry: rapid, calibrated measurements of cell mechanical properties. Biophys J. 2017;113(7):1574-84 Epub 2017/10/06.

Otto O, Rosendahl P, Mietke A, Golfier S, Herold C, Klaue D, et al. Real-time deformability cytometry: on-the-fly cell mechanical phenotyping. Nat Methods. 2015;12(3):199-202 4 p following Epub 2015/02/03.

Park SY, Gonen M, Kim HJ, Michor F, Polyak K. Cellular and genetic diversity in the progression of in situ human breast carcinomas to an invasive phenotype. J Clin Invest. 2010;120(2):636-44 Epub 2010/01/27.

Patel AP, Tirosh I, Trombetta JJ, Shalek AK, Gillespie SM, Wakimoto H, et al. Single-cell RNA-seq highlights intratumora heterogeneity in primary glioblastoma. Science. 2014;344(6190):1396-401 Epub 2014/06/14

Pawlizak S, Fritsch AW, Grosser S, Ahrens D, Thalheim T, Riedel S, et al. Testing the differential adhesion hypothesis across the epithelial- mesenchymal transition. New J Phys. 2015;17(8):083049.

Perez-Moreno M, Fuchs E. Catenins: keeping cells from getting their signals crossed. Dev Cell. 2006;11(5):601-12.

Plodinec M, Loparic M, Monnier CA, Obermann EC, Zanetti-Dallenbach R, Oertle P, et al. The nanomechanical signature of breast cancer. Nat Nanotechnol. 2012;7(11):757-65 Epub 2012/10/23.

Remmerbach TW, Wottawah F, Dietrich J, Lincoln B, Wittekind C, Guck J. Oral cancer diagnosis by mechanical phenotyping. Cancer Res. 2009:69(5):1728-32 Epub 2009/02/19.

Schmidt B, Kießling T, Warmt E, Fritsch A, Stange R, Käs JA. Complex thermorheology of living cells. New J Phys. 2015;17(7): 073010 .

Schnauß J, Händler T, Käs JA. Semiflexible biopolymers in bundled arrangements. Polymers. 2016;8(8):274.

Shackleton M, Quintana E, Fearon ER, Morrison SJ. Heterogeneity in cancer: cancer stem cells versus clonal evolution. Cell. 2009;138(5):822-9 Epub 2009/09/10.

Strehle D, Schnauss J, Heussinger C, Alvarado J, Bathe M, Kas J, et al. Transiently crosslinked F-actin bundles. Eur Biophys J. 2011:40(1):93-101 Epub 2010/08/25.

Swaminathan V, Mythreye K, O'Brien ET, Berchuck A, Blobe GC, Superfine R. Mechanical stiffness grades metastatic potential in patient tumor cells and in cancer cell lines. Cancer Res. 2011;71(15):5075-80 Epub 2011/06/07.

Taketo MM. Reflections on the spread of metastasis to cancer prevention. Cancer Prev Res (Phila). 2011;4(3):324-8 Epub 2011/ 03/05.

Torres L, Ribeiro FR, Pandis N, Andersen JA, Heim S, Teixeira MR. Intratumor genomic heterogeneity in breast cancer with clonal divergence between primary carcinomas and lymph node metastases. Breast Cancer Res Treat. 2007;102(2):143-55 Epub 2006/08/15.

Wirtz D, Konstantopoulos K, Searson PC. The physics of cancer: the role of physical interactions and mechanical forces in metastasis. Nat Rev Cancer. 2011;11(7):512-22 Epub 2011/06/28.

Xu W, Mezencev R, Kim B, Wang L, McDonald J, Sulchek T. Cell stiffness is a biomarker of the metastatic potential of ovarian cancer cells. PLoS One. 2012;7(10):e46609.

Zhu G, Yan HH, Pang Y, Jian J, Achyut BR, Liang X, et al. CXCR3 as a molecular target in breast cancer metastasis: inhibition of tumor cell migration and promotion of host anti-tumor immunity. Oncotarget. 2015;6(41):43408.

\section{Publisher's Note}

Springer Nature remains neutral with regard to jurisdictional claims in published maps and institutional affiliations. 